\title{
Circuitos Socio-Asistenciales para Población en Situación de Calle en la Ciudad de Buenos Aires: Representaciones Sociales y Prácticas
}

\section{Socio-Care Circuits for Homeless People in Buenos Aires' City: Social Representations and Practices}

\author{
Jorgelina Di Iorio, Susana Seidmann, Gustavo Rigueiral y Yamila Abal \\ Universidad de Buenos Aires
}

\begin{abstract}
El presente trabajo se enmarca en un proyecto de investigación participativa financiado por la Universidad de Buenos Aires, que tuvo como objetivo indagar las trayectorias de vida y los procesos de construcción de identidad en personas en situación de calle (PSC) en la Ciudad de Buenos Aires, Argentina, y generar un espacio de participación y reflexión colectiva que facilite problematizar dichas trayectorias, así como los circuitos socio-asistenciales por los que transitan. Con una modalidad de investigación cualitativa de tipo participativo, el trabajo se organizó en 3 etapas: (a) diagnóstico participativo, (b) problematización y (c) primera intervención. En forma intencional, se conformó una muestra de 10 organizaciones que son parte de la oferta asistencial a las PSC, y con un muestreo bola de nieve una de 18 PSC adultas usuarias de esta oferta, hombres y mujeres, que se encuentran en situación de calle con una permanencia mayor a 3 años. Por medio de entrevistas, grupos focales, un mapeo colectivo y la construcción de un árbol de problemas, y utilizando el enfoque de la teoría fundamentada en los hechos y análisis de contenido, se encontró que las intervenciones se organizan en función de ciertas representaciones sociales sobre la situación de calle, sobre las propias personas y sobre lo que se entiende por "atender el problema". A partir de estos resultados, se problematizan los límites y desafíos de las intervenciones con enfoques comunitarios con PSC, así como la relación entre la universidad y la comunidad en los procesos de construcción de conocimientos.
\end{abstract}

Palabras clave: situación de calle, intervención psicosocial, participación

\begin{abstract}
This work is part of an action-research funded by the University of Buenos Aires. Its aims were to investigate the life trajectories and the processes of identity construction in homeless people in the City of Buenos Aires, Argentina, and create a space for participation and collective reflection that facilitates the problematization of both these trajectories and socio-care circuits for homeless people. Using participative qualitative research, this work had three stages: (a) participatory diagnosis, (b) problematization and (c) first intervention. An intentional sample was selected of 10 organizations that were part of the healthcare offer for homeless people, and with a snowball sampling one of 18 homeless adults users of this offer, both men and women, who were in the streets for at least 3 years. Through interviews, focus groups, collective mapping, and the construction of a problem tree, and using grounded theory approach, it was found that interventions are organized according to certain social representations of the street situation, of the people themselves and of what is meant by "addressing the problem". Based on these results, the limits and challenges of interventions with community-based approaches with homeless people are problematized, as well as relations between the University and the Community in the process of building knowledge.
\end{abstract}

Keywords: homeless people, psychosocial interventions, participation

Siguiendo a Castel (2004), las transformaciones societales producto del modo en que economías concentradas y transnacionalizadas impactaron, durante los años 70, en la organización del orden social, dio lugar a la configuración de zonas de integración-vulnerabilidad-expulsión en la vida social. Poniendo el foco en la pérdida del trabajo como soporte de inscripción privilegiado, tanto en los países latinoamericanos y del

Jorgelina Di Iorio, Susana Seidmann, Gustavo Rigueiral y Yamila Abal, Instituto de Investigaciones, Facultad de Psicología, Universidad de Buenos Aires, Argentina.

El presente artículo presenta algunos resultados del proyecto de investigación UBACyT 20020130100089BA, financiado por la Universidad de Buenos Aires, el cual se desarrolló en el período 2014-2017. El proyecto contó con el aval del Comité Ad Hoc de Conductas Responsables en Investigación de la Facultad de Psicología de la Universidad de Buenos Aires. Al momento de esta publicación, el proyecto se encuentra finalizado con el informe final en repositorios virtuales. Por tal motivo, algunos de los fragmentos de este artículo pueden encontrarse en esas publicaciones digitales, en particular lo que hace referencia a la descripción metodológica y la presentación de algunos resultados. Este artículo corresponde a la publicación de la ponencia realizada en el XXXV Congreso Interamericano de Psicología que se llevó a cabo en Lima, Perú (Seidmann, Di Iorio, Rigueiral \& Abal, 2015, Julio).

La correspondencia relativa a este artículo debe ser dirigida a Jorgelina Di Iorio, Instituto de Investigaciones, Facultad de Psicología, Universidad de Buenos Aires, Gral. Juan Lavalle 2353, Ciudad Autónoma de Buenos Aires, CP 1052AAA, Argentina. E-mail: diiorio.jorgelina@gmail.com 
Caribe, como en el resto del mundo, emergen esas zonas en función de una doble desafiliación socio-laboral (Bustelo \& Minujín, 1997; Castel, 2004).

Como producto de la profundización de los procesos de segmentación social y la poca movilidad social, se cristalizan en las grandes ciudades zonas de pobreza urbana, en las que grupos sociales constituidos desde su pertenencia a un todo social fragmentado tienen un acceso desigual a bienes, servicios y derechos (Kessler \& Merklen, 2013). Al momento de la escritura de este artículo, en Argentina se cumplían seis meses de gestión del gobierno del PRO, que a diferencia del gobierno del período 2003-2015 implantó un modelo económico que se tradujo en aumento de la inflación, devaluación de la moneda, despidos masivos y aumento de impuestos. El estancamiento de la actividad económica y la restricción y vulneración de derechos generaron un aumento de la pobreza, registrándose 1,4 millón más de pobres que en 2015 (Pontificia Universidad Católica Argentina, 2016). Es decir, se configura una forma particular de opresión caracterizada por la vulneración sistemática de derechos. Entre estas desigualdades durables (Clemente, 2012), entendido en términos de un continuum exclusióninclusión (Bustelo \& Minujín, 1997) o de inclusión desde la marginalidad (Sawaia, 2011), se incluye la situación de calle como un modo en que se expresan los procesos de expulsión social en los grandes centros urbanos.

Describir el circuito socio-asistencial para personas en situación de calle (PSC), distinguiendo obstáculos y facilitadores de las intervenciones, tanto desde el punto de vista técnico como ético, permite problematizar el modo en que se conceptualiza el problema y las prácticas de intervención que se despliegan. En este sentido, el articulo pretende, a partir de la presentación de un proceso de investigación participativa y problematizar las lógicas de intervención con PSC, con la intención de diseñar dinámicas de trabajo que promuevan transformaciones subjetivas y colectivas, así como la ampliación de derechos.

\section{Personas en Situación de Calle: Definir un Campo de Problemas}

En la literatura internacional, se encuentran diferentes categorías que hacen referencia a este campo de problemas. Mientras que algunas ponen el acento en la dimensión residencial o falta de vivienda para definir el problema, recurriendo a términos como sinhogarismo (Federación Europea de Organizaciones Nacionales que trabajan con las personas sin hogar, 2008), otras ponen el acento en la dimensión vincular. Es decir, en los sentidos y usos particulares del espacio público y las dinámicas de reconocimiento y diferenciación social (Boy, 2012; Di Iorio, Seidmann, Gueglio \& Rigueiral, 2016; Rosa, 2010).

En este sentido, estar en situación de calle se define como una problemática social compleja caracterizada por un continuo proceso de posesión/desposesión material, simbólico y afectivo. La problemática no se reduce a quienes utilizan literalmente el espacio público como lugar de pernocte. Incluye a todo otro conjunto de personas que utilizan la red de alojamientos nocturnos transitorios - hogares y refugios- y quienes se encontrarían en riesgo de situación de calle. Constituyen una de las formas en las que se institucionalizan los procesos de vulneración, caracterizados por diferencias económicas, desigualdades jurídicas y desafiliaciones sociales, que se traducen en la vulneración de derechos (Di Iorio et al., 2016).

La calle, en tanto término polisémico, implica abrigo, es decir, el lugar donde se está alojado, y modo de vida, en tanto compleja red de relaciones que se torna invisible para el conjunto de la sociedad. Desde esta perspectiva, estar en situación de calle no es un estado o una cosa, sino una relación social, donde lo efímero se convierte en constante, emergiendo una forma de padecimiento social relacionada con expresiones de inequidad e injusticia social, así como también se hacen poseedores de atributos socialmente desacreditadores, dando lugar a procesos de estigmatización (Goffman, 1963/2003). La profundización de la distancia social entre estos grupos sociales y el resto de la sociedad da lugar a que sean vistos como grupos socialmente amenazantes, culturalmente estigmatizados y económicamente marginales.

En la Ciudad de Buenos Aires, según los datos oficiales, se estima que hay aproximadamente 1096 PSC, mientras que las organizaciones sociales y comunitarias hablan de 7251, según el Segundo Censo Popular de Personas en Situación de Calle (CPPSC), que fue realizado en abril de 2019, en compañía del Ministerio Público de la Defensa de la Ciudad de Buenos Aires, con la intención de confrontar el número oficial. Con una metodología participativa y un diseño territorial de amplio alcance se recorrieron los 48 barrios de la ciudad (CPPSC, 2019). En 2017, las mismas organizaciones realizaron el Primer Censo Popular, frente a la negativa del gobierno local de convocar a una mesa de diálogo y trabajo intersectorial. Para ampliar sobre estos relevamientos, ver Di Iorio (2019a) y Di Iorio y Farías (2019, Octubre).

Pese a lo que pudiera considerarse desde una mirada ingenua, la cotidianeidad de las PSC se caracteriza por el despliegue de un conjunto de secuencias prestablecidas temporal y espacialmente para satisfacer 
necesidades básicas (Di Iorio et al., 2016). La cronificación de esta situación de vida genera que las PSC se inscriban simbólicamente desde lo que les falta, recorriendo una territorialidad organizada a partir de circular por distintas ofertas socio-asistenciales. Dicha oferta socio-asistencial está integrada por (a) organizaciones confesionales; (b) gubernamentales, (c) sociales y/o políticas; (d) no gubernamentales no confesionales y (e) grupos autoconvocados, que a los fines de este estudio se definieron como dispositivos. Por dispositivo se entiende un conjunto heterogéneo de elementos, en los que se incluyen individuos ejerciendo distintos roles, con uno o varios objetivos en común, que surgen para atender un problema o una situación, desplegados en un tiempo particular, que "se implementan para una cierta población e implican la utilización de diferentes formas de encuadre de trabajo, requisitos y normas de funcionamiento, horarios, formas de contener, de escuchar y de orientar" (Pawlowicz et al., 2011, p. 177). Desde ese conjunto de dispositivos se despliegan prácticas de intervención psicosocial que tienen como finalidad el mejoramiento de las condiciones de vida de las PSC o la promoción de su bienestar social. Es la población con la que trabajan lo que hace que los equipos definan sus prácticas como comunitarias, poniendo el acento en el objeto de intervención más que en las dimensiones técnicas y ético-políticas de las mismas (Di Iorio, 2019b).

\section{Entre Usuarios y Dispositivos: Representaciones Sociales y Prácticas}

En esos escenarios de interacción circulan significaciones y valoraciones entre quienes ofrecen determinados servicios y quienes concurren, convirtiéndose las PSC en objeto de representación social (RS). Las RS constituyen sistemas de interpretación que rigen la relación con el mundo y con los otros, orientando y organizando las experiencias de la vida cotidiana (Jodelet, 1984/1986). Conforman categorías que permiten clasificar, interpretar y dar sentido a la vida cotidiana, cobrando especial relevancia en su elaboración el contexto y la vivencia de los actores sociales involucrados, lo cual constituye un saber experiencial. Es decir, hay una relación entre el modo en que determinado objeto es definido - $\mathrm{RS}$ - y las prácticas o acciones que se despliegan entre esos sujetos, existiendo entre RS y prácticas relaciones de interdependencia y transformación recíproca. Esas prácticas, que se configuran en formas institucionales específicas (dispositivos) y las RS que las condicionan, están ancladas en relatos o narrativas sociales legitimados histórica y culturalmente. Esos relatos organizan lo decible, lo narrable y lo pensable; se establecen como valor de verdad y operan como instrumentos ideológicos.

Las RS se configuran como aprendizajes sociales situados que se negocian, se apropian y se transforman en los escenarios de interacción cotidiana. Es decir, no son abstracciones, sino que están enraizadas, ancladas de manera situada (Di Iorio, 2019b). La vida colectiva se caracteriza por su forma narrativa, lo que permite la organización y la comunicación de experiencias: "con el tiempo, el compartir historias comunes crea una comunidad de interpretación (...) una narración modela no sólo un mundo, sino también las mentes que intentan darle sus significados" (Bruner, 2003, pp. 45-47).

En el marco de una investigación participativa (Sirvent, 2011/1999) de tipo exploratorio-descriptivo, que tuvo como objetivos generales indagar las trayectorias de vida y los procesos de construcción de identidad en PSC en la Ciudad de Buenos Aires (Argentina) y generar un espacio de participación y reflexión colectiva que facilitara problematizar sus recorridos de subsistencia, surgieron los siguientes interrogantes que se pretenden responder en el artículo y que permiten caracterizar el circuito socio-asistencial: ¿Qué caracteriza el tipo de vínculo que establecen las PSC con esos dispositivos? ¿Qué definiciones - RS — sobre las PSC circulan? ¿Qué tipo de prácticas de intervención configuran? ¿Qué lugares identitarios emergen? ¿En qué relatos sociales están ancladas?

\section{Método y Resultados}

\section{Diseño: el Rol Central de la Participación}

Como plantea Wiesenfeld (2014), la psicología social comunitaria se caracteriza por priorizar la participación, la reflexión crítica y la concientización sobre los modos en que opera el orden social hegemónico como forma de promover el cambio social. Implica el desplazamiento desde una mirada psicológica individual hacia la social-comunitaria, adoptando como valores la inclusión, el respeto a la diversidad de experiencias, saberes y recursos y la democratización de las relaciones de poder.

Desde esa perspectiva, el proyecto en el que se incluye este trabajo combina técnicas convencionales de investigación social, tales como entrevistas en profundidad, observación participante, técnicas gráficas y 
asociación libre de palabras, con otras técnicas más participativas, dialógicas y dinámicas, que incluyen a los/as participantes en su autoestudio (Montero, 2004): mapeos colectivos, juegos dramáticos y sesiones de retroalimentación. La participación, en el sentido del involucramiento activo de los/as participantes, no implica un método de trabajo, sino la valoración y el rescate de lo que es propio de los actores involucrados, su historia, su experiencia, en el intento de trabajar de una forma contextualizada. Tal como sostiene Sánchez Vidal (1991), la participación es "un valor, una técnica y un proceso" (p. 272).

Entendiendo que la investigación participativa es una práctica social de producción de conocimientos que busca la transformación social y que el conocimiento científico se produce en la propia acción al mismo tiempo que contribuye a ella, el trabajo de campo se organizó en distintas etapas: (a) diagnóstico participativo, (b) problematización y (c) primera intervención. Esto dio lugar a un proceso recursivo de construcción de sentidos organizado a partir de la secuencia reflexión-descripción-acción-reflexión. Entre cada etapa se realizó una sesión de retroalimentación con los/as participantes. Si bien la etapa de diagnóstico constituye de algún modo un nivel de problematización, se diferencia de la segunda porque en esta última se promovió el trabajo colectivo de discusión, mientras que en la primera se focalizó en la descripción de las relaciones entre las prácticas de asistencia y las RS sobre situación de calle sobre las que se fundan.

\section{Procedimientos de Rigor}

En pos de garantizar la validez de los datos recolectados, a lo largo de todo el proceso de investigación se realizaron como procedimientos de rigor: (a) cotejo e integración de los datos encontrados con documentaciones y trabajos previos, (b) criterio de saturación conceptual que implicó la confirmación a partir de la reiteración de conceptos/categorías construidas (Berteaux, 1993, citado en Callejo, 1998) y (c) sesiones de retroalimentación (Sirvent, 2011/1999).

Se realizaron tres sesiones de retroalimentación (Sirvent, 2011/1999), una después de cada una de las etapas - diagnóstico, problematización, intervención-, que tuvieron como finalidad el análisis crítico de los problemas centrales identificados, promoviendo la reducción de la distancia entre las prioridades de investigación y las necesidades de la población implicada. En esta línea, se distinguen tres momentos/sentidos de las sesiones de retroalimentación, que se presentan de manera cronológica: (a) presentación de la información obtenida y sistematizada por el equipo de investigación luego de la etapa de diagnóstico; (b) proceso reflexivo-creativo de los y las participantes, constituidos/as en grupos de trabajo luego de la etapa de problematización y (c) surgimiento de nuevas propuestas para la investigación en el marco de la etapa de intervención. Cada sesión se constituyó en un espacio de convergencia de diferentes fuentes de información; en este sentido, puede ser considerado como situaciones de triangulación metodológica en situ (Sirvent, 2018). Los mismos sirvieron para construir y validar el conocimiento, promover la capacidad de la población de objetivar la realidad cotidiana, compartir y discutir la información con actores sociales involucrados en la temática, desarrollar técnicas de trabajo colectivo y discutir los resultados.

\section{Consideraciones Éticas}

Tomando la dimensión interpersonal y el reconocimiento como eje central de la ética en investigación e intervención desde la psicología social comunitaria, los/as participantes fueron informados/as vía oral y vía consentimiento informado de los objetivos del proyecto, así como de los resultados, a través de sesiones de retroalimentación que se llevaron a cabo al finalizar cada una de las etapas. Asimismo, se conservaron las normas de confidencialidad y derecho a la intimidad, pudiendo los/as participantes dejar de participar en cualquier momento del proceso.

\section{Análisis de Datos}

El análisis de los datos se realizó bajo el enfoque de la teoría fundamentada en los hechos (Strauss \& Corbin, 1998), en combinación con análisis de contenido (Bardin, 1977/1986). Fueron efectuados análisis de tipo axial y selectivo. La codificación axial se realizó a partir de categorías emergentes surgidas de una primera categorización abierta de los datos, identificando las variaciones (dimensiones) de las mismas. Esas categorías se reintegraron y refinaron a partir del establecimiento de relaciones entre las mismas, identificándose al par sujeto de derecho-objeto de intervención como categoría central explicativa (codificación selectiva). 
En el caso de la etapa de diagnóstico, las entrevistas, las transcripciones y la primera codificación fueron realizadas por el equipo de investigación y legitimadas por PSC e integrantes de instituciones de referencia. En la etapa de problematización, se trató de promover la toma de consciencia por parte de los y las participantes, a través de un proceso de construcción colectiva de conocimientos. Además, se promovió la lectura crítica de la vida cotidiana y se inició un proceso de redefinición de necesidades colectivas y diseño de propuesta de intervención (etapa de primera intervención).

\section{Etapa 1: Diagnóstico Participativo}

Objetivo específico. Describir la problemática de las PSC en la zona sur de la Ciudad de Buenos Aires.

Participantes. La muestra fue teórica intencional por criterios (Patton, 1990). Participaron cinco PSC y 10 referentes de organizaciones que conforman el circuito socio-asistencial. Para la selección de PSC se recurrió a un muestreo de bola de nieve (Patton, 1990), es decir, cada entrevistado/a sugirió a otro participante. Como criterios de inclusión se consideraron hombres, mujeres y personas trans, mayores de 18 años, que usaran los servicios asistenciales de la zona sur de la Ciudad de Buenos Aires y que estuvieron hace más de tres años en situación de calle. Para la muestra de referentes se realizó un listado preliminar de organizaciones localizadas en la zona sur de la Ciudad de Buenos Aires, que fueron identificadas en investigaciones previas (Programación UBACyT 2007-2011, 2011-2014) por las PSC como parte de la oferta asistencial. Se garantizó la distribución por cuotas de pertenencia institucional — confesional, dependencia gubernamental, dependencia no gubernamental, organización comunitaria, vecinos autoconvocados(categorización construida en estudios previos, ver Di Iorio et al., 2016), que los/as participantes fueran parte de los equipos técnicos, sin distinción de profesión, y que tuvieran una antigüedad mínima de un año en la tarea. Las instituciones participantes se eligieron en función del criterio de accesibilidad.

Instrumento. Se realizaron entrevistas en profundidad, tanto a los referentes como a las PSC, para caracterizar las formas institucionales que adquiere la atención psicosocial para esta población, describir las RS que se construyen sobre las PSC e identificar en qué relatos socioculturales esas RS se anclan. La guía de las entrevistas incluyó los siguientes temas: definición de la problemática, génesis de la situación de calle, relación asistido-asistente y actividades e implicación personal. Las entrevistas fueron grabadas y luego transcritas en formato digital para su análisis computarizado. Se realizaron en lugares consensuados con las personas que aceptaron participar, previo consentimiento informado.

Resultados. En los relatos de los referentes se identificó que las PSC son ubicadas como objetos receptores de cuidado, constituyendo lo que se conoce como ciudadanía asistida. Se diferencian definiciones centradas en lo que falta, desde procesos del des (desafiliado, desenganchado, desempleado) y aquellas centradas en lo que se debe hacer, desde procesos del re (reinsertar, reintegrar, reeducar, reafiliar) (ver Tabla 1).

Tabla 1

Objetivación de la RS sobre PSC: Contenido-Definiciones

\begin{tabular}{|c|c|}
\hline Desde el des & Desde el re \\
\hline $\begin{array}{l}\text { Una persona que está en la calle es porque perdió todo, su } \\
\text { trabajo, su familia, no tiene nada (...) está por fuera de } \\
\text { todo. (voluntaria, organización confesional) }\end{array}$ & $\begin{array}{l}\text { si querés salir de la calle tenés que aprender todo de nuevo, } \\
\text { desde cómo comer hasta el uso del baño (...) cómo te tratan, } \\
\text { reaccionan mal (...) es como volver a empezar (...) y eso } \\
\text { hacemos, volvés a enseñar (operador de calle, dependencia } \\
\text { gubernamental) }\end{array}$ \\
\hline $\begin{array}{l}\text { Es el conjunto societalmente excluido que vive en o de la } \\
\text { calle como única opción. Incluye, entonces, tanto a la gente } \\
\text { que circula y vive de la calle, como los cartoneros, como } \\
\text { también a quienes están alojados en paradores, en } \\
\text { hospedajes precarios a los que accedieron a través de un } \\
\text { subsidio habitacional, a chicas y chicos en calle (...) son los } \\
\text { que nadie ve, invisibles (...) desaparecidos sociales. } \\
\text { (voluntaria, dependencia no gubernamental no } \\
\text { confesional) }\end{array}$ & $\begin{array}{l}\text { En la calle tenés libertad, no te llega una carta ni un } \\
\text { impuesto, nada, entonces las intervenciones tienen que ver } \\
\text { con la reinserción, con que se esté incluido, y empezás } \\
\text { muchas veces con el documento. (psicóloga, dependencia } \\
\text { gubernamental) }\end{array}$ \\
\hline
\end{tabular}


Sobre los motivos explicativos de la situación de calle, si bien se reconoce la complejidad de la problemática y su multidimensionalidad, se diferenciaron dos tipos de explicaciones: las que se focalizan en aspectos de la historia individual y familiar (asociadas a separaciones, violencias, fallecimientos y otras problemáticas de lo familiar, padecimientos mentales y consumos problemáticos de sustancias psicoactivas), en contraposición a explicaciones centradas en condicionamientos socio-históricos (flexibilidad del mercado de trabajo, pobreza persistente, consumo de drogas como problema social, marginalización social) (ver Tabla 2).

Tabla 2

Objetivación de las RS sobre PSC: Contenido y Génesis de la Situación de Calle

\begin{tabular}{|c|c|}
\hline Individual-familiar & Socio-histórico \\
\hline $\begin{array}{l}\text { hombres que se separaron, se van de la casa, perdieron el } \\
\text { trabajo, no tienen dónde ir. Hay muchos hombres así, que } \\
\text { vos los ves que tratan de conservar su higiene y que andan } \\
\text { dando vueltas... que no tienen por ahi una red familiar o } \\
\text { de amigos que lo puedan contener y de repente cayó en } \\
\text { calle. O les da vergüenza pedir ayuda y están en calle por } \\
\text { ese motivo. Mujeres que también quedaron solas, se } \\
\text { separaron. Jubilados, muchos jubilados, que no les } \\
\text { alcanza y están en la calle. (voluntaria, grupo } \\
\text { autoconvocado comunitario) }\end{array}$ & $\begin{array}{l}\text { Todo ese contexto [refiriéndose a la fragmentación y } \\
\text { marginalización social] de alguna se inscribe en la } \\
\text { subjetividad de ese sujeto. Ese sujeto hablante, vos lo } \\
\text { escuchas, te transmite todo eso. Se presenta y se presenta } \\
\text { así, se presenta como pobre, feo, temeroso, amenazante. } \\
\text { (trabajadora social, dependencia gubernamental) }\end{array}$ \\
\hline $\begin{array}{l}\text { El juego, el alcohol, las drogas... y también mucha gente } \\
\text { que ha venido de afuera y ha tenido algún inconveniente } \\
\text { delictivo o pasó algo y perdieron contacto con su familia. } \\
\text { (voluntaria, mujer, organización confesional) }\end{array}$ & $\begin{array}{l}\text { No tiene que ver únicamente con los recursos materiales o } \\
\text { algo económico, lo que tienen o no estas personas, sino } \\
\text { como el resultado de un proceso de una sociedad } \\
\text { excluyente. (psicóloga, mujer, organización no } \\
\text { gubernamental) }\end{array}$ \\
\hline
\end{tabular}

Dicho argumento sobre necesitar cuidados también se desprende de los relatos de las PSC, quienes se refieren a sí mismas como aquellas que no pueden, que tienen problemas, que no tienen ganas, que son irresponsables. Esas percepciones sobre sí mismas, producto de la negociación intersubjetiva de significados, se sostiene en atribuciones que estigmatizan. Se configura como núcleo figurativo de la RS una imagen de PSC definida desde la lógica del déficit, habilitando la intervención psicosocial para recuperar aquello perdido. De alguna manera, esto hace invisibles procesos de reafiliación o de configuración de nuevos vínculos sociales que, tal como relatan las PSC, son generados por las propias personas, de alguna manera independientemente de la intervención de los equipos.

Se registró que quienes ponen el acento en definiciones de la PSC centradas en el des, el tipo de relación que establecen con las PSC es de dependencia y asistencialista, mientras que para quienes la definición está centrada en el re, el vínculo se orienta hacia la autonomía y empoderamiento (ver Tabla 3).

Se distinguieron dos lógicas de intervención: una tutelar y otra restitutiva. Desde la lógica tutelar, la relación se basa en una otredad distante fundada en una ética moralista del deber ser. Desde la lógica restitutiva, la relación se configura desde una subjetividad próxima, basada en una ética relacional. Específicamente, la dimensión ética

reside en el reconocimiento y la aceptación del Otro en su diferencia, en su aceptación como sujeto cognoscente con igualdad de derechos (...) en la intersubjetividad que al reconocer la humanidad del Otro permite que, por ese acto, el Uno también sea humano. (Montero, 2004, p. 106)

Y de esa relación, que sea liberadora o no, depende la construcción de autonomía o reproducción de las condiciones de exclusión. Resulta interesante que, pese a sus diferencias, desde ambas lógicas se coloca a las PSC como receptoras de cuidado, específicamente, desplegando prácticas focalizadas en la dimensión material del cuidado, que pretende cubrir necesidades de vestido, higiene, alimentación y pernocte. Utilizando la saturación conceptual como herramienta de validación (Strauss \& Corbin, 1998) se analizaron los significados sobre PSC, diferenciando en función del tipo de institución. No se encontró una asociación entre el tipo de institución —religiosa, comunitaria, gubernamental—y la lógica de intervención — tutelar o restitutiva一, así como tampoco en la dimensión de contenido o núcleo figurativo de la RS de PSC, cristalizada 
en la imagen de "necesitar cuidados". Sin embargo, al analizar los modos en que ese cuidado se hace operativo (las prácticas de intervención), se registró en los referentes entrevistados de las organizaciones sociales y/o políticas el predominio de una ética relacional, a diferencia de los de las confesionales, las ONG's, los grupos autoconvocados y las organizaciones gubernamentales, en los que predominan discursos moralizantes.

Tabla 3

Objetivación de las RS sobre PSC: Contenido-Tipo de Vínculo entre PSC y Dispositivos

\begin{tabular}{ll}
\hline Autonomía/Empoderamiento & \multicolumn{1}{c}{ Dependencia/Asistencialismo } \\
\hline $\begin{array}{l}\text { En el comedor popular que forma parte de la asamblea... } \\
\text { atiende a gente en calle los domingos... con una modalidad }\end{array}$ & $\begin{array}{l}\text { gente en situación de calle como una problemática } \\
\text { muy autogestiva, son las mismas personas en situación de }\end{array}$ \\
calle quitacional, entonces, sus politicas y los programas están \\
con un rol fuertemente activo por parte de ellos. (militante \\
social, organización social y/o política) & $\begin{array}{l}\text { aparecen allí los subsidios o paradores; pero eso constituye } \\
\text { ya de por sí una limitación. Yluego, también, por parte de }\end{array}$ \\
& $\begin{array}{l}\text { las iglesias se piensa como una ayuda caritativa para } \\
\text { paliar esta situación, por lo que termina reforzando estos } \\
\text { círculos, estos circuitos donde las personas circulan } \\
\text { mecánicamente por estos espacios, reproduciendo la }\end{array}$ \\
& $\begin{array}{l}\text { situación de exclusión y la situación de calle. (psicóloga, } \\
\text { organización no gubernamental no confesional) }\end{array}$
\end{tabular}

El objetivo de nuestro proyecto es facilitar el acceso a la salud en las personas en situación de calle, garantizar de alguna forma que ese derecho a la salud no esté vulnerado. Creemos que esta es una función que tiene que cumplir el Estado (psicóloga, organización no gubernamental no confesional)

\section{Etapa 2: Problematización}

Objetivo específico. Problematizar los saberes y prácticas sobre la vida cotidiana de las PSC y describir obstáculos, tensiones y desafíos en el acceso a bienes, servicios y derechos, desde su propia perspectiva.

Participantes. La muestra, que fue no probabilística intencional de bola de nieve o red (Patton, 1990), se conformó con 15 PSC, 10 hombres y cinco mujeres, de una edad promedio de 40 años, que utilizan los servicios de asistencia en la zona sur de la Ciudad de Buenos Aires y que tienen más de tres años en situación de calle. Esta técnica de muestreo, basada en el conocimiento y/o acercamiento de las PSC, es eficaz en el trabajo con población en condición de extrema vulnerabilidad social, en tanto facilita la construcción de vínculos de confianza entre el investigador y los/as participantes. Solo dos de las personas que participaron de la primera etapa se incluyeron en las sesiones de mapeo. Eso no debe comprenderse en términos de superposición con la etapa anterior, sino como estrategia para garantizar la participación en esta segunda instancia. Las PSC se definen como población oculta o difícil en función de ser considerada rara, en el sentido estadístico (poco frecuente, geográficamente concentrada o dispersa), ser oculta (por comportamientos ilegales o especiales) o fluctuante (intermitencia de su asociación a puntos concretos del espacio geográfico) (Bastos \& Bertoni, 2014). Que la invitación a participar la realizaran personas que participaron de la etapa anterior facilitó la construcción de vínculo de confianza con el equipo a cargo de la investigación.

Instrumentos. Se realizó un mapeo colectivo. Mapear es una estrategia que permite, de manera simultánea, construir conocimientos e intervenir sobre la realidad social (Risler \& Ares, 2013). En un proceso dialéctico, el territorio construye subjetividades y, a su vez, es definido y cargado de sentido desde las propias simbolizaciones y relatos de quienes lo habitan. Es una herramienta que permite abordar territorios geográficos y subjetivos, tomando como principio orientador la participación (Di Iorio, Rigueiral, Gueglio \& Abal, 2015). Para esto, se realizaron dos sesiones de un grupo focal, con posterioridad a la sesión de retroalimentación, en las que se socializaron y discutieron los resultados del diagnóstico participativo. Se 
utilizaron distintas técnicas recreativas para promover la interacción entre los/as participantes, tales como teatro espontáneo y otros juegos dramáticos, escritura colaborativa y disparadores audiovisuales.

Resultados. Vivir en la calle en contexto urbano, según los/as participantes, se configura como un campo temático organizado en tres planos o capas de sentido, tal como se muestra en la Figura 1.

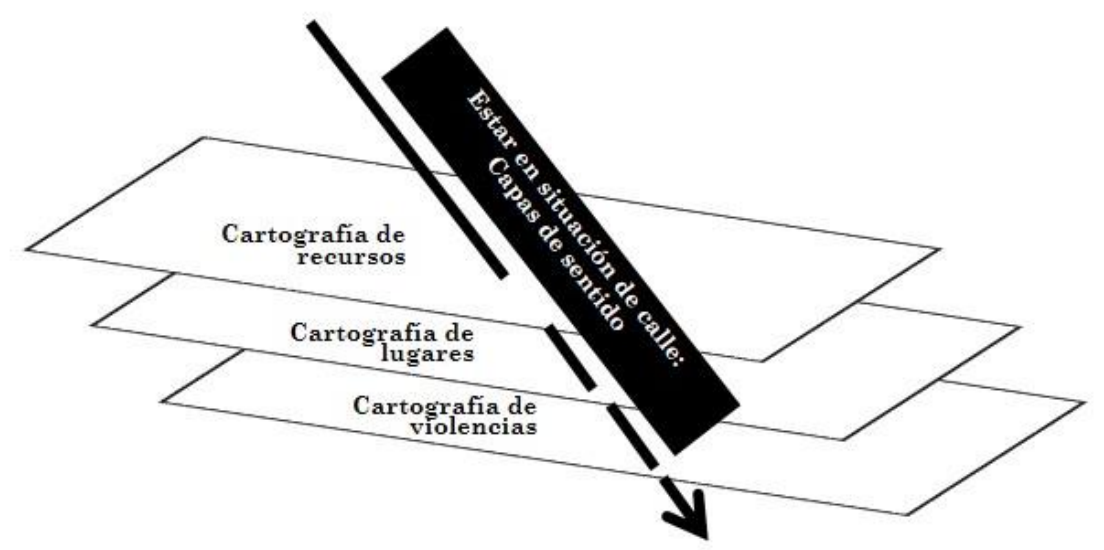

Figura 1. Planos de sentido sobre la situación de calle.

Cartografía de recursos. La distribución de servicios y actividades alude a la descripción de todo el circuito socio-asistencial. Los/as participantes acordaron en que la oferta se organiza en función de la satisfacción de las necesidades básicas, agregando que hay organizaciones en las que pueden realizar actividades educativas, tanto para terminar estudios formales como para capacitarse en oficios (electricidad, plomería, panadería, serigrafía). También se incluyó en esta categoría lo que los/as participantes llamaron actividades para ganarle horas al día, aludiendo a distintos tipos de talleres recreativos a los que concurren, no tanto por el contenido que se aborda, sino para reducir situaciones de violencia y/o consumo de drogas que se dan en el espacio público y para ampliar sus intercambios afectivos y simbólicos. En la Figura 2 se observa esa distribución de ofertas.

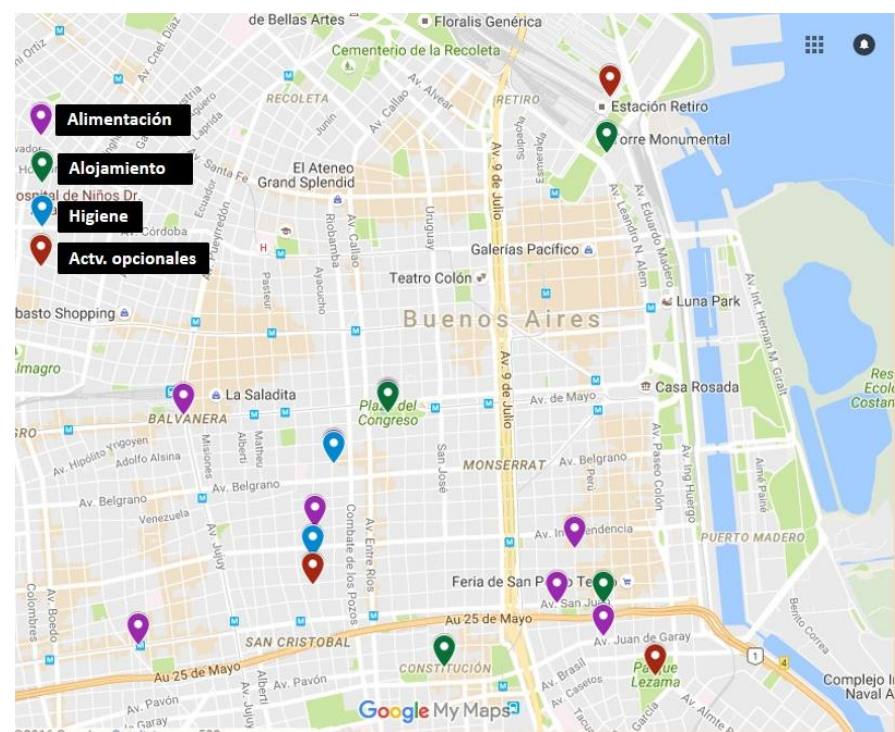

Figura 2. Oferta socio-asistencial en la zona sur de la Ciudad de Buenos Aires. 
Se configura una rutina de subsistencia organizada a partir de la percepción de un uso distribuido del espacio y una circularidad temporal, en la que se reconoce la base normativa desde la que se construye el espacio público. Es decir, en función de los sentidos atribuidos, se registran usos diferentes del espacio según grupo social, los cuales se califican como adecuados o inadecuados en base a los criterios social y culturalmente atribuidos. De algún modo, ese argumento es el que legitima el control social sobre el espacio urbano (Berroeta Torres \& Vidal Moranta, 2012).

Cartografía de lugares. Se identificó en los relatos sobre las trayectorias cotidianas de los/as participantes referencias a lugares de la ciudad, en particular plazas o parques, que son en los que desarrollan la mayoría de sus actividades. Es decir, espacios públicos significativos de la Ciudad de Buenos Aires, donde las personas establecen relaciones entre su historia personal y ese lugar (ver Figura 3).

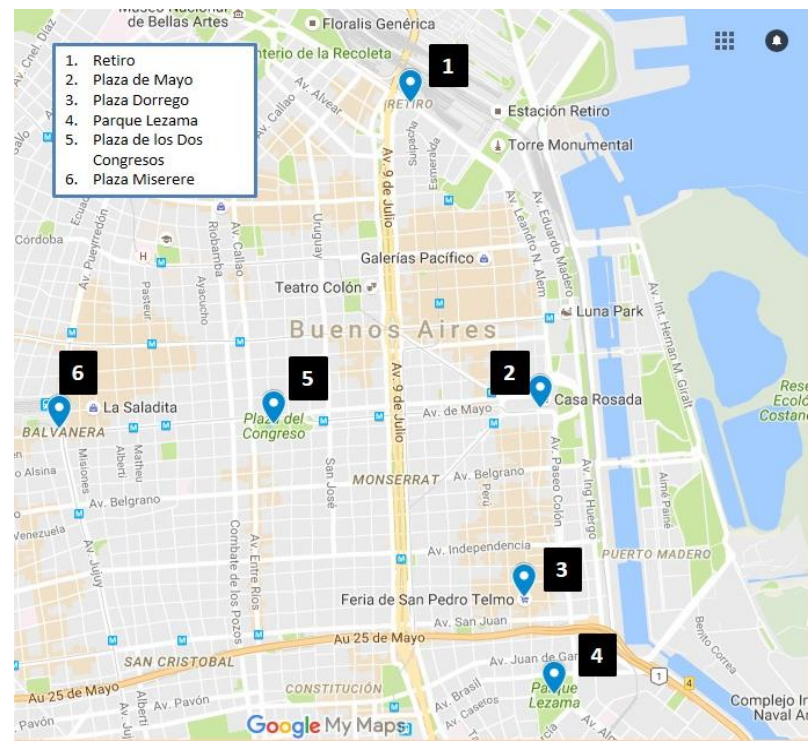

Figura 3. Localización de lugares públicos (plazas) en la Ciudad de Buenos Aires.

Estos lugares públicos se caracterizan por la interacción esporádica o específica para la población en general y de interacción continua y escenario de múltiples intercambios sociales, afectivos, económicos, políticos y culturales para las PSC. Ellos se pueden revisar en la Tabla 4.

Tabla 4

Plazas de la Ciudad Diferenciada por Uso y Sentido

\begin{tabular}{lll}
\hline \multicolumn{1}{c}{ Lugar de la Ciudad } & \multicolumn{1}{c}{ Uso socialmente establecido } & \multicolumn{1}{c}{ Uso por las PSC } \\
\hline Plaza de los dos Congresos & Actividad administrativa & Alojamiento \\
& & Uso de drogas \\
& & Alimentación \\
& & Supervivencia* \\
Plaza de Mayo & Movilizaciones sociales y políticas & Alojamiento \\
& Turismo & Resistencia \\
Retiro & Terminal de micros de larga distancia & Actividades opcionales** \\
& Actividades comerciales & Alojamiento \\
Plaza Miserere & Terminal de trenes & Alimentación \\
& Actividades comerciales & Actividades opcionales \\
Parque Lezama & Turismo & Actividades opcionales \\
Plaza Dorrego & Turismo & Actividades opcionales \\
& & Subsistencia
\end{tabular}

* Actividades de supervivencia alude a las acciones legales e ilegales para conseguir dinero.

** Actividades opcionales se refieren a actividades de ocio (lectura, ajedrez, encuentros, talleres). 
Lo que se desprende es que no se puede hablar de la Ciudad en un sentido homogéneo, sino que, como nudo de significaciones, es representada en función de cómo es habitada.

Cartografía de violencia. A medida que los/as participantes identificaron lugares públicos e instituciones como parte del circuito socio-asistencial, describieron valoraciones positivas o negativas en relación con las personas y/o las actividades que allí se despliegan. Mayoritariamente, las PSC relatan situaciones de discriminación y destrato, como si algo de su condición de vida se convirtiera en un atributo desacreditador susceptible de convertirse en un estigma (Goffman, 1963/2003). Refieren que, en la mayoría de los servicios, las PSC se sienten desechables y como si quisieran que se vayan rápido. Asimismo, enuncian que son pocos o nulos los espacios en los que sienten que se les escucha, es decir, que "son más que un plato de comida". Expresan situaciones de malestar y de angustia y la falta de espacios amigables donde poder contar lo que les pasa. Se registran situaciones de violencia física entre pares y también por parte de las fuerzas de seguridad en el espacio público.

\section{Etapa 3: Primera Intervención}

No es el objetivo de este artículo la presentación en profundidad del diseño e implementación de la intervención, sino explicitar esta última como momento de un tipo de investigación social.

A partir de los resultados de la segunda etapa y de lo discutido en una segunda sesión de retroalimentación, en la que participaron cinco PSC que participaron de la etapa 2 , referentes de una de las organizaciones comunitarias que participó de las dos etapas anteriores (Asociación Civil Asamblea Popular Plaza Dorrego-San Telmo, localizada en el barrio de San Telmo, en la zona sur de la Ciudad de Buenos Aires) y miembros del equipo de investigación, se construyó un árbol de problemas en el que se identificaron problemas centrales y secundarios, así como causas y consecuencias, en función de las vivencias en la comunidad (Ver Figura 4).

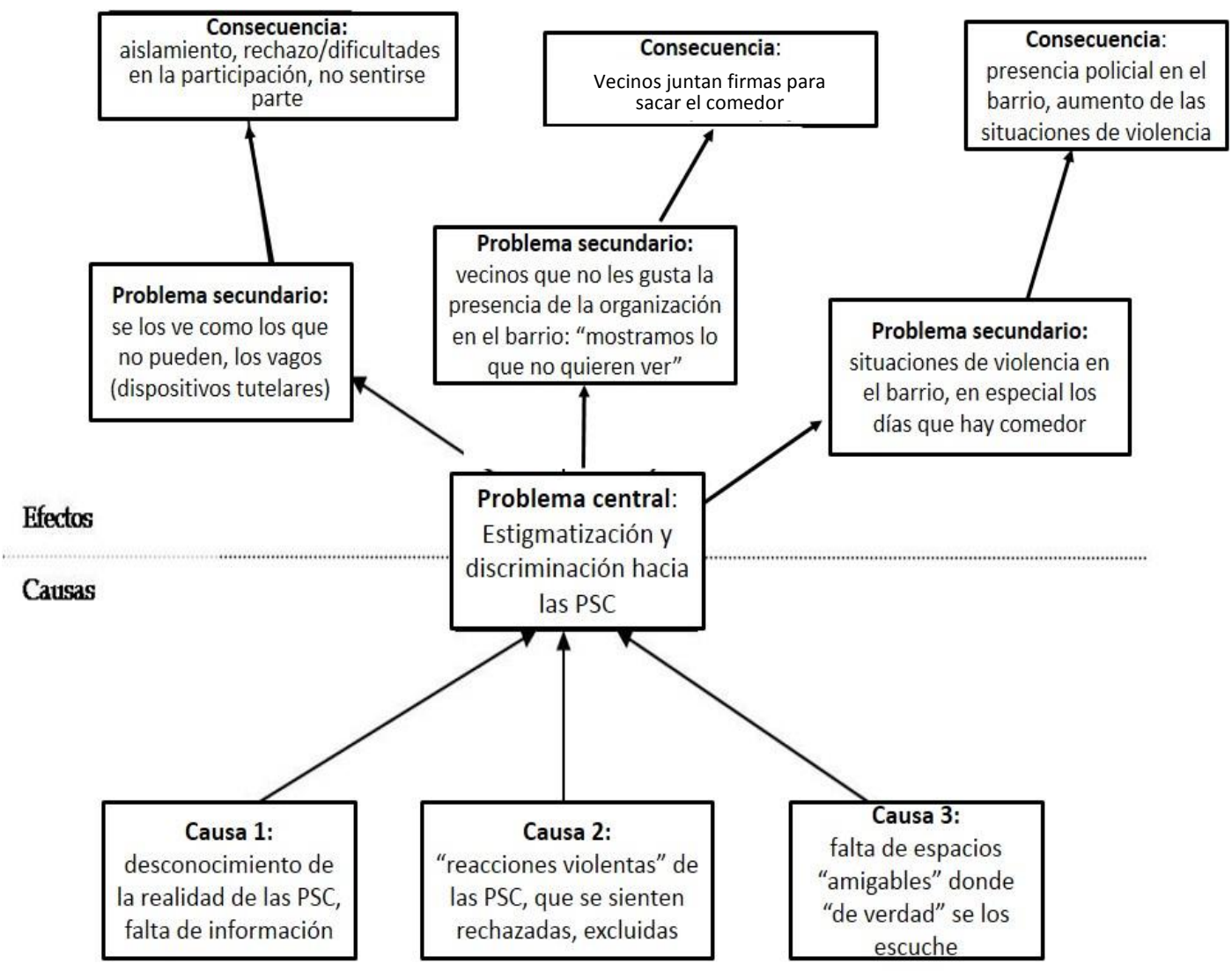

Figura 4. Árbol de problemas: planificación de la intervención. 
Se puso en evidencia que estar en situación de calle se convierte en un atributo desacreditador, no solo para quienes están en esa situación, sino también para las organizaciones que ofrecen algún tipo de asistencia para esta población. En este sentido, y en función de los problemas delimitados, se diseñó de manera participativa un trabajo en colaboración, organizado en dos líneas: (a) con las propias PSC a partir de fortalecer el espacio de encuentro para PSC que funciona en la organización participante ("Malabardeando... hacemos malabares para dejar el bardo") y (b) con la comunidad, a partir de actividades abiertas.

En lenguaje coloquial, el término bardo es utilizado por las PSC para referirse a situaciones conflictivas, problemas o excesos que tienen consecuencias negativas para las personas. Específicamente, la expresión hacemos malabares para dejar el bardo habilita a las PSC a incluirse como sujetos activos en la gestión de riesgos y la reducción de daños, a partir de la socialización de estrategias, así como también en la construcción colectiva de prácticas de cuidado y autocuidado. Por malabares se entiende al arte de manipular y ejecutar espectáculos con uno o más objetos a la vez, volteándolos, manteniéndolos en equilibrio o arrojándolos al aire alternativamente, usualmente sin dejar que caigan al suelo. Los malabares se conocen por su dificultad y por la necesidad de poseer ciertas habilidades. La iniciativa de los concurrentes al comedor que participaron de la etapa 2 y de las sesiones de retroalimentación, sobre la necesidad de un espacio de escucha, se traduce en la actualidad en un dispositivo de salud comunitaria, que fue cogestionado entre la Facultad de Psicología, Universidad de Buenos Aires y la Asociación Civil Asamblea Popular Plaza Dorrego-San Telmo, pero que en la actualidad solo se desarrolla desde la asociación civil. Basado en el reconocimiento de las PSC como sujetos, se pretende recuperar su condición socio-histórica, promover la exigibilidad de derechos y mejoras en la calidad de vida, atendiendo a la singularidad de los padecimientos. Este espacio se materializa en encuentros semanales de una hora y media de duración, con una dinámica fluida en el diálogo e intercambio, antes del almuerzo los días domingo. Si bien es un grupo abierto y tiene rotación de participantes, participan 12 miembros estables. El grupo se configuró como un ámbito propicio para la desnaturalización de las RS sobre estar en situación de calle, orientado a la resignificación de sus experiencias, con el propósito de generar transformaciones subjetivas y colectivas con un sentido emancipador. Se pretende superar la dinámica tutelar que predomina en el circuito socio-asistencial y la vivencia de "falta de espacios amigables y de escucha" que expresaron las PSC, promoviendo la construcción de otros posicionamientos sociales sobre la base de la participación, que, como dice Sánchez (2000), implica "tener parte de" algo que pertenece a un grupo, "tomar parte en" algo con otros y "ser parte de" algo, involucrarse.

En lo que respecta a la segunda línea de trabajo, desde el grupo de apoyo se planifican actividades abiertas a la comunidad. Con una frecuencia mensual, los participantes organizan un ciclo de expresión artística y una radio abierta. En ambas actividades se promueve la participación al comedor de otros concurrentes que no participan activamente del grupo, pero que gradualmente se van incorporando a otras actividades de la organización, así como también de otras personas de la comunidad que no están en situación de calle. Estos escenarios de interacción integrados reducen la distancia y se traducen en una disminución del estigma social.

\section{Discusión}

A partir de los resultados presentados, surgen dos nuevos interrogantes en relación con los límites de las intervenciones psicosociales y comunitarias en el campo de las marginaciones sociales: ¿Implica el retorno de argumentos que culpabilizan e inhabilitan o supone problematizar el lugar de los sujetos en la gestión de sus afecciones o padecimientos? ¿Las intervenciones psicosociales son comunitarias? ¿Son lo mismo? ¿Qué aportes para el desarrollo de la psicología social comunitaria se desprenden de este trabajo?

Describir las lógicas que sostienen el sistema socio-asistencial para PSC supuso indagar el modo en que este sistema impacta sobre el desarrollo de alternativas de integración social, así como también sobre los límites de las intervenciones y la banalización de lo que se entiende por comunitario. De los resultados, se desprende que, en su mayoría, las intervenciones con PSC están al servicio de una reducción de daños o de mejorar el bienestar psicosocial, siendo limitada la promoción de la participación y su inclusión activa en la gestión de sus propios padecimientos. Mayoritariamente, las prácticas que se describieron previamente están mediadas por RS sobre las PSC basadas en el descontrol, la irresponsabilidad y la incapacidad, lo que, de alguna manera, inhabilita considerarlas sujetos políticos, capaces de incluirse activamente en procesos de transformación comunitaria. Siguiendo a Rose (2007), esas formas de entender, clasificar y actuar sobre las PSC, en tanto que sujetos de gobierno, tienen implicancias no solo en las prácticas de los equipos técnicos y referentes institucionales, sino también en las que las propias personas implementan para gobernarse a sí 
mismas. Desde esta perspectiva, las PSC son consideradas como no afiliadas a ninguna colectividad en virtud de su incapacidad para gestionarse a sí mismas como sujetos, o se los considera afiliados a algún tipo de "anticomunidad" cuya moralidad, estilo de vida o comportamiento se consideran una amenaza o un impedimento" (Rose, 2007, p. 130).

Sin embargo, también se observaron intervenciones que, sostenidas desde una lógica restitutiva de derechos más que tutelar, se focalizan en

la necesidad de la participación de las personas, en el apoyo a sus cualidades positivas y en el fomento de sus capacidades, es decir, en el fortalecimiento de esos individuos y grupos para que logren por sí mismos transformaciones positivas que mejoren su calidad de vida y su acceso a bienes y servicios de la sociedad a la cual pertenecen (Montero, 2003, p. 59)

pero de la que son continuamente expulsados. Son prácticas de resistencia en las que se les concede una nueva relación activa con su situación, en términos de sus estrategias y capacidades para la gestión de sí mismos, reconociéndolos como sujetos de derechos y como sujetos políticos.

Sin desconocer las secuelas y el impacto que las vulneraciones sistemáticas de derechos, producidas por décadas de neoliberalismo, han tenido en la construcción de identidades y en los procesos de subjetivación fundamentalmente en determinados colectivos sociales, interesa resaltar que incluso en las condiciones sociales más hostiles el hombre no deja de ser un sujeto con su historia, sus prácticas, sus relaciones, sus sentimientos y sus pensamientos. Hacer hincapié en estos aspectos es, por un lado, un intento de no reproducir miradas que cosifican y, por otro, pretende ser un aporte que sirva para avanzar en los procesos de subjetivación de esta población. Los relatos de los/as participantes dan cuenta del modo en el que los encuentros semanales propician la problematización y desnaturalización de aspectos de sus trayectorias. Frente a la configuración de identidades estigmatizadas, se hacen visibles subjetividades que resisten, cuerpos que desafían ser negados y negativizados, sujetos que tienen un saber sobre sus padecimientos y que ponen en funcionamiento prácticas fundadas en la solidaridad, el respeto y la dignidad. En este sentido, intervenciones que promuevan la participación comunitaria, así como también el diseño de acciones que promuevan la exigencia de derechos, se alejan de la mera producción y/o ampliación de bienestar social. Es decir, pasarían de intervenciones psicosociales a intervenciones comunitarias.

Finalmente, en lo que respecta a las contribuciones de este trabajo al desarrollo de la psicología social comunitaria, se abre un campo de indagación en lo que dice relación con la distinción entre intervenciones psicosociales, en el sentido de promover el bienestar social y fundadas en los desarrollos de una psicología social más clásica, y las intervenciones comunitarias, ligadas a la promoción de la participación y a la construcción de autonomía en contextos de vulneraciones de derechos. Se retoma en este punto la distinción que realiza Berroeta (2014) en su reflexión sobre las formas que adquiere el quehacer de la psicología comunitaria.

En el mismo sentido, retomando a Prilleltensky y Nelson (2002), se hace necesario distinguir si la intervención -y también la investigación - dentro de la psicología comunitaria genera opresión o emancipación, no solo en función de sus resultados, sino del proceso en sí mismo. La dimensión política adquiere central relevancia, ya que el poder no es algo que afecta de tal o cual manera a las poblaciones en condiciones de vulnerabilidad a las que se interviene, sino que el poder es constitutivo de las prácticas de investigación e intervención y se introduce en la forma en que se piensa acerca de las personas con las que se trabaja y en la forma en la que son tratadas. Es decir, se usa poder para investigar e intervenir sobre el poder.

Por lo tanto, el desafío para el quehacer profesional, así como para el desarrollo teórico y metodológico de la disciplina y para las intervenciones con marginaciones sociales en particular, lo constituye reflexionar sobre las prácticas empleadas e indagar sobre sus efectos, incorporando la problematización sobre las relaciones de poder, desde las cuales se configuran relaciones de dependencia o de autonomía.

\section{Referencias}

Bardin, L. (1977/1986). Análisis de contenido (C. Suarez, Trad.; Título original: The Content Analysis). Madrid, España: Akal.

Berroeta, H. (2014). El quehacer de la psicología comunitaria: coordenadas para una cartografía. Psicoperspectivas, 132 , 19-31. https://doi.org/10.5027/psicoperspectivas-Vol13-Issue2-fulltext-352

Berroeta Torres, H. \& Vidal Moranta, T. (2012). La noción de espacio público y la configuración de la ciudad: fundamentos para los relatos de pérdida, civilidad y disputa. Polis, 31, 1-21. https://doi.org/10.4067/S0718-65682012000100004

Boy, M. (2012). Adultos que viven en la calle: políticas públicas, usos y estrategias en torno a la ciudad. Buenos Aires, 1997-2011. Tesis de doctorado no publicada. Universidad de Buenos Aires, Facultad de Ciencias Sociales, Argentina.

Bruner, J. (2003). La fábrica de historias. Derecho, literatura, vida. Buenos Aires: Fondo de Cultura Económica. 
Bustelo, E. \& Minujín, A. (1997). La política social esquiva. En R. Menjívar Larín, D. Kruijt \& L. van Vucht Tijseen (Eds.), Pobreza, exclusión y política social (pp. 113-154). San José, Costa Rica: Facultad Latinoamericana de Ciencias Sociales.

Callejo, J. (1998) Los límites de la formalización de las prácticas cualitativas en investigación social: la saturación. Sociológica: Revista de Pensamiento Social, 3, 93-119. Extraído de https://core.ac.uk/download/pdf/61895053.pdf

Castel, R. (2004). Las trampas de la exclusión. En R. Castel (Ed.), Las trampas de la exclusión: trabajo y utilidad social (pp. 21-38). Buenos Aires, Argentina: Topía.

Clemente, A. (2012). Pobreza persistente. Una problemática poco explorada. En Eudeba, Universidad de Buenos Aires \& PIUBAMAS (Comps.), Universidad y políticas públicas: el desafío ante las marginaciones sociales (pp. 401-412). Buenos Aires: Eudeba

Censo Popular Personas en Situación de Calle (2019). Segundo censo popular de personas en situación de calle en la caba. Extraído de https://www.cels.org.ar/web/2019/07/segundo-censo-popular-de-personas-en-situacion-de-calle-en-la-caba/

Di Iorio, J. (2019a). Vivir en situación de calle en contextos urbanos: subjetividades en resistencia. Revista Interamericana de Psicología, 53, 167-179. https://doi.org/10.30849/rip/ijp.v53i2.1067

Di Iorio, J. (2019b). ¿Cómo cuidar sin tutelar? Notas sobre un modelo de intervención en contextos de vulneraciones psicosociales basado en los vínculos. En E. Nelson Arellano (Ed.), Situaciones de calle. Abandonos y sobrevivencias. Miradas desde las praxis: Chile Argentina - Costa Rica - México (pp. 25-47). Santiago, Chile: RIL.

Di Iorio, J. \& Farías, M. (2019, Octubre). Mapear la violencia y la exclusión en Buenos Aires: la experiencia del Censo Popular de Personas en Situación de Calle. Ponencia presentada en el $4^{\circ}$ Congreso Latinoamericano de Estudios Urbanos, Universidad Nacional General Sarmiento, Los Polvorines, Argentina. Extraído de https://www.academia.edu/40493776/Mapear_la_violencia_y_la_exclusi\%C3\%B3n_en_Buenos_Aires_la_experiencia_del_Censo_P opular_de_Personas_en_Situaci\%C3\%B3n_de_Calle

Di Iorio, J., Rigueiral, G. J., Gueglio, C. \& Abal, Y. (2015). Construyendo colectivamente mapas y territorios (poster). En V. S. Piatti (Comp.), Memorias del $5^{\circ}$ Congreso Internacional de Investigación de la Facultad de Psicología de la Universidad Nacional de La Plata (pp. 546-548). Buenos Aires, Argentina: Universidad Nacional de La Plata.

Di Iorio, J., Seidmann, S., Gueglio, C. \& Rigueiral, G. (2016). Intervenciones psicosociales con personas en situación de calle: el cuidado como categoría de análisis. Psicoperspectivas, 15(3), 123-134. https://doi.org/10.5027/psicoperspectivas-Vol15-Issue3-fulltext-838

Federación Europea de Organizaciones Nacionales que trabajan con las personas sin hogar (2008). El papel de la vivienda en el Sinhogarismo. Alojamiento y Exclusión residencial. Informe Europeo. Extraído de https://www.feantsa.org/download/08_european_report_feantsa_housing_final_es7074115848578375806.pdf

Goffman, E. (1963/2003) Estigma: la identidad deteriorada. Buenos Aires: Amorrortu.

Jodelet, D. (1984/1986). La representación social: fenómenos, conceptos y teoría (D. Rosenbaum, Trad.; Título original: Représentations sociales: phénomènes, concept et théorie). En S. Moscovici (Comp.), Psicología social, II. Pensamiento y vida social: psicología social y problemas sociales (Título original: Psychologie Sociale) (pp. 478-494). Barcelona, España: Paidós.

Kessler, G. \& Merklen, D. (2013). Una introducción cruzando el Atlántico. En R. Castel, G. Kessler, D. Merklen \& N. Murard (Cords.), Individuación, precariedad, inseguridad. ¿Desinstitucionalización del presente? (pp. 9-31). Buenos Aires, Argentina: Paidós.

Montero, M. (2003). Teoría y práctica de la psicología comunitaria: la tensión entre comunidad y sociedad. Buenos Aires, Argentina: Paidós.

Montero, M. (2004). Introducción a la psicología comunitaria: desarrollo, conceptos y procesos. Buenos Aires, Argentina: Paidós.

Patton, M. Q. (1990). Qualitative evaluation and research methods (2a ed.). Thousand Oaks, CA: SAGE.

Pawlowicz, M. P., Galante, A., Goltzman, P., Rossi, D., Cymerman, P. \& Touzé, G. (2011). Dispositivos de atención para usuarios de drogas: heterogeneidad y nudos problemáticos. En Argentina, Ministerio Público Tutelar, Panorámicas en salud mental: a un año de la sanción de la Ley Nacional N 26.657 (pp. 169-188). Buenos Aires, Argentina: Editorial Universitaria de Buenos Aires.

Pontificia Universidad Católica Argentina (2016). Informe Empleo, Precariedad Laboral y Desigualdades Estructurales en la Argentina Urbana. Buenos Aires.

Prilleltensky, I. \& Nelson, G. (2002). Doing psychology critically: Making a difference in diverse settings. New York, NY: Palgrave Macmillan.

Risler, J. \& Ares, P. (2013). Manual de mapeo colectivo: recursos cartográficos críticos para procesos territoriales de creación colaborativa. Buenos Aires, Argentina: Tinta Limón.

Rosa, P. (2010). El circuito de la necesidad: Las Organizaciones de la Sociedad Civil y la asistencia a los habitantes de la calle en la Ciudad Autónoma de Buenos Aires. Summer School on Interdependent Inequalities in Latin America Brazil, 1-5.

Rose, N. (2007). ¿La muerte de lo social? Re-configuración del territorio de gobierno. Revista Argentina de Sociología, 5(8), 111-150. Extraído de http://www.redalyc.org/articulo.oa?id=26950807

Sánchez, E. (2000). Todos con la "esperanza": continuidad de la participación comunitaria. Caracas, Venezuela: Universidad Central de Venezuela, Facultad de Humanidades y Educación, Comisión de Estudios de Postgrado.

Sánchez Vidal, A. (1991). Psicología comunitaria. Bases conceptuales y operativas: métodos de intervención. Barcelona, España: Promociones y Publicaciones Universitarias.

Sawaia, B. (Org.) (2011). As artimanhas da exclusão: análise psicossocial e ética da desigualdade social [Las artimañas de la exclusión: análisis psicosocial y ética de la desigualdad social] (14 ed.). Petrópolis, Brasil: Vozes.

Seidmann, S., Di Iorio, J., Rigueiral, G. \& Abal, Y. (2015, Julio). Circuitos Socio-Asistenciales para Población en Situación de Calle en la Ciudad de Buenos Aires: Representaciones Sociales y Prácticas. Ponencia presentada en el XXXV Congreso Interamericano de Psicología, Lima, Perú.

Sirvent, M. T. (2011/1999). Cultura popular y participación social. Una investigación en el barrio de Mataderos (2da reimpresión). Buenos Aires, Argentina: Miño y Dávila

Sirvent, M. (2018). De la Educación Popular a la Investigación Acción Participativa. Perspectiva pedagógica y validación de sus experiencias. InterCambios. Dilemas y transiciones de la Educación Superior, 5(1), 12-29. https://doi.org/10.29156/inter.5.1.10

Strauss, A. \& Corbin, J. (1998). Basics of qualitative research: Techniques and procedures for developing grounded theory (2a ed.). Thousand Oaks, CA: SAGE.

Wiesenfeld, E. (2014). La psicología social comunitaria en América Latina: ¿consolidación o crisis? Psicoperspectivas, $13(2), 6-18$. https://doi.org/10.5027/psicoperspectivas-Vol13-Issue2-fulltext-357.

Fecha de recepción: Junio de 2017.

Fecha de aceptación: Diciembre de 2019. 